\title{
Thomas Blanke \\ 75 Jahre Betriebsverfassung: Der Siegeszug eines historischen Kompromisses
}

\author{
1. Das Betriebsrätegesetz von 1920: "Aberratio ictus" in dic richtige \\ Richtung?
}

Das Betriebsrätegesetz vom 4.2. 1920 war ein Quantensprung in der Entwicklung des deutschen Arbeitsrechts. In ihm bündelte sich das Zusammenwirken von Elementen traditionaler, nationalspezifischer »Industriekultur" und krisenhaft-revolutionärer Auseinandersetzung in einem Modell von Betriebsverfassung, das für Deurschland bis heute prägend geblieben ist. Das geltende, inzwischen mehrfach novellierte Betriebsverfassungsgesetz vom i s. x . 1972 führt ebenso wie sein unmittelbarer Vorläufer vom Ix.10.1952 die mit dem Betriebsrätegesetz vor 70 Jahren erstmals entwickelten Strukturen, die aus einer eigentümlichen Mixtur von Betriebsfriedens- und Betriebsgemeinschaftsideen und gekappten rätedemokratischen Konzepten hervorgegangen sind, konsequent und um echte Mitbestimmungsrechte bereichert fort. So umstritten die seinerzeit gefundene Betriebsverfassung auch war (und in so gut wie allen Details noch ist), so sehr besteht doch inzwischen in der bundesdeutschen Debatte Einigkeit darüber, daß dieser historische Kompromiß im Prinzip in die richtige Richtung weist: In Richtung auf die gleichberechtigte Mitwirkung der Arbeitnehmer in Betrieb und dic Zuerkennung eines betrieblichen »Bürgerstatus«, der den Schutz der Persönlichkeit des Arbeitnehmers und seiner Grundrechte ernst nimmt.'

\footnotetext{
I Dic Dcutung des politischen Entwicklungsprozesses als Abfolge schrittweise errungener Staatsbürgerrechte (personlichc Frciheitsrechte, politische Kommunikationsrechte, demokratische Mit- und Selbstbestimmungsrechte, soziale Teilhaberechte) ist bekannt und wetthin ublich. Auch der Betriebswverfassung*, die die herrschende Rechtsauffassung der Weimarer Zeit (vgl. Urteil des RAG v. 10. 7. 1929, Bensh. Samml. Bd. VI, S. 4Os) noch zum offentlichen Recht (!) zahlte, liegt ebenso wie der Konzeption der Wirtschaftsdemokratie die Vorstellung zugrunde, daß der betriebliche weithin rechtlose Status der Arbeitnehmer in cin paralleles konstututionelles, burgerschaftlich-demokratisches Rechtsverhaltunis als "Arbeitsburger (E. Fraenkel, Zehn Jahre Betricbsrategesetz, wiederabgedruckt in: T.Ramm (Hrsg.), Arbeitsrecht und Politik. Quellentexte 1918-1933, 1966, S. 97 ff., I 11 ) zu überführen sei. Die Herausarbeitung dieser Paral. lelentwicklung steht im Zentrum der Untersuchung von Karl Korsch, Arbeitsrecht fur Betriebsrate, aus dem Jahr 1922 (Neuauflage Frankfurt 1968) und gipfelt in der Prognose; s So gewiß nun in der Entwicklung des politischen Gemeinwesens der Konstitutionalismus nur eine Úbergangsphase zwischen absoluter Monarchie und voller burgerbche Demokratie gewesen ist, so gewiß wird auch in der Entwicklung der Verfassung des Gemeinwesens der Arbeit auf die heute noch nicht einmal vollsiandig entwickelte, sondern noch ganz in ihren Kinderschuhen steckende Phase des sgewerblichen Konstitutionalismuss eine Phase volliger industrieller Demokratiec enmal folgen mussene, a. a. O. S. s1. Dic in der aktuellen soziologischen und rechelichen Debatte beobachtbare Wiederaufnahme dieses Konzepts stutzt sich bemerkenswerter Weise nicht auf diese sepzifisch deutsche Tradition, sondern auf die spate Rezeption von Arbeiten, insbesondere von T. H. Marshall, Citizenship and Social Class, in: dgl., Sociology at the Crossroads and other Essays, London 1963, S. I ff.; deutsch: Staatsburgerrechte und soziale Klassen, in: dgl., Bürgerrechte und soziale Klasse. Zur Soziologie des Wohlfahrtsstaates, Frankfurt/M. 1992, S. 33 ff., die in der industriellen Wirklichkeıt Großbritanniens deutlich wenıger empirische Bestatigung finden. Dieser wissenschaftliche Rückimport « ist eigentumlich und erklärungsbedurftig. Móglicherweise liegt die Erklarung darin, daß sich die Demoksaticthcorie in der angloamerikanischen Diskussion anders als in der kontinentaleuro-
} 
Die Entstehung des Betriebsrätegesetzes war überschattet von blutigen Ereignissen: Wenige Tage zuvor wurden bci einer Demonstration vor dem Reichstagsgebäude in Berlin anläßlich der Lesung des Betriebsrätegesetzes in der verfassunggebenden Deutschen Nationalversammlung 42 Arbeiter von Militär und Polizei durch Maschinengewehrfeuer und Handgranaten getötet. Die Verantwortung für dieses Blutbad übernahm der preußische Innenminister Heine (SPD), Reichspräsident Ebert, ebenfalls SPD, verhängte über weite Teile des Reiches den Ausnahmezustand. Kein Zweifel: Das Betriebsrätegesetz von 1920 wurde gegen den erbitterten, ja verzweifelten Widerstand von erheblichen Teilen der Arbeiterschaft verabschiedet. Daß dieses Gesetz gleichwohl strukturbildend für die Entwicklung eines modernen, auf die Demokratisierung der betrieblichen Herrschaft des Arbeitgebers zielenden Arbeitsrechts werden sollte, stand ihm bei seiner Geburt nicht auf die Stirn geschrieben.

Wie kaum ein zweites Ereignis in der Geschichte der deutschen Arbeiterbewegung wirft die Entstehung der Betriebsverfassung ein düsteres Licht auf die Dialektik des Rechrsfortschritts im Arbcitsrecht - was sich heute im distanzierten historischen Rückblick als fortschrittlich, gar richtungsweisend erweist, mußte von den Betroffenen damals, zumal den engagiertesten unter ihnen, als Verrat an ihren viel weitergehenden revolutionären Erwartungen wahrgenommen werden. Vor dem Hintergrund der welthistorischen Alternative Kapitalismus oder Sozialismus erschien das Betriebsrätegesetz von 1920 zwangsläufig als fauler Kompromiß zugunsten des ökonomischen Status quo, allenfalls Jegitimierbar in der Perspektive eines kontinuicrlich weitergehenden Rechtsfortschritts. Der gedankliche Fluchtpunkt solcher Systemtransformation hat sich inzwischen aufgelöst, ist Utopie geworden. Dies verändert den Blick auf die Geschichte der Reformen in kapitalistischen Gesellschaften und der von ihnen ausgelösten Lern- und Veränderungsprozesse: Wo es an dem für realistisch gehaltenen Ziel des "ganz andercn « fehlt, crgibt sich die Notwendigkeit, den geschichtlichen Innovationen einen eigenständigen Sinn abzugewinnen und ihn nicht länger an eine Geschichtsteleologie zu delegieren, die ihre Geschichte hinter sich hat. Dabei wird sich herausstellen, daß auch die Arbeitsrechtsgeschichte längst Entwicklungspfade eingeschlagen hat, die mit den Wegweisungen der Protagonisten ihrer Haupt- und Staatsaktionen und ihrer ideologischen Wortführer wenig gemein haben. ${ }^{2}$

passchen Debatte weitgehend unabhangig von der ideologischen Uberdeterminierung durch die sozialistische Geschichtsphilosophte entfaltet hat. Demokratue erhalt so eınen normatıven Eigensınn, der sich nicht aus der Vision einer finalen Aufhebung gesellschaftlicher Konflikte speist, sondern in threr Überfuhrung in ratıonale, diskursıve Verfahren der Normbegrundung, Interessenlegitimation und Konfliktaustragung. Der Renaissance der Diskussion um den Burgerstatus im Betrieb entspricht auf gesamtgesellschaftlicher die Debatte uber das - verwandte - Konzept der "Zivilgesellschaft", welches ebenfalls aus der angloamerikanischen Diskussion ubernommen wurde, vgl. U. Rodel, G. Frankenberg, H. Dubiel, Dic demokratische Frage, 1991. Zur gegenwartigen Debatte um Burgerrechte im Betrieb vgl. W. Muller-Jentsch, Uber Produktivkräfte und Burgerrechte, in: Sozıale Welt Sonderband 9, 1994 S. 643 ff.; H. Kotchoff, Betriebsrate und Burgerstatus, 1994; H. Matthies, U. Mückenberger, C. Offe, E. Peter, S. Raasch, Arbeit 2000. Anforderungen an eine Neugestaltung der Arbeststvelt, 1994.

2 Vgl. hierzu auch T. Blanke. Die Entdeckung des Arbeitsrechts durch die Gewerkschaften, ArbuR 1994, S. $113 \mathrm{ff}$. 


\section{Betriebsverfassung und nationale Arbeitsrechtskultur: Die Wurzeln des Betriebsrätegesetzes von 1920}

Stärker als andere Rechtsgebiete ist das Arbeitsrecht von nationalen Traditionen und den Besonderheiten ihrer gcschichtlichen Entwicklung geprägt: Deshalb fällt es den Mitglicdsstaaten der Europäischen Union so schwer, sich auf einheitliche Strukturen eines europäischen Arbeitsrechts zu einigen. Dies gilt insbesondere für die Betriebsverfassung. Dic hierzu unternommenen Ansätzc ciner Vereinheitlichung sind bislang immer wieder gescheitert, obwohl sie schon darauf verzichteten, einen bestimmten Typus der Betriebsverfassung verbindlich vorzuschreiben und statt dessen ein »Menue « möglicher Gestaltungsformen zur Auswahl stellen. ${ }^{3}$ Die in diesem Jahr verabschiedete Richtlinie über die Betriebsräte in europaweit operierenden Unternchmen ist angesichts dessen nicht mehr als ein erster, schwacher Lichtstreifen am Horizont künftiger Rechtseinheit. ${ }^{4}$

Die Gründe für die nationale Prägung des Arbeitsrechts sind vielfältig: Sie liegen einerseits in überkommenen kulturspezifischen Ordnungsvorstellungen, dic in den Formen der Organisation und den sozialen Strukturen der industriellen Produktion zum Ausdruck kommen. Andererseits schlägt sich in der Entwicklung des Arbeitsrechts - deutlicher als selbst im Verfassungsrecht - die Geschichte der sozialen Auseinandersetzungen, der in ihrem Verlauf cntstandenen Organisationen und ihrer politischen Ideologien und Strategien nieder. Das Arbeitsrecht ist immer auch cin Lesebuch zur Sozialgcschichtc cines Landes mit ihren Konflikten, Siegen, Niederlagen und Kompromissen. Daher ist es auch seinem Selbstverständnis nach typischerweise ein unabgeschlossen, offenes Projekt. Nicht zufällig mangelt es in fast allen Ländern an einer gesetzlichen Regelung des Arbeitskampfrechts. Oft beruht, wie in Deutschland, auch das Individualrecht nicht auf einer geschlossenen Kodifikation, sondern stellt ein unübersichtliches Kompendium gesetzlicher Einzelregelungen dar, die z. T. bis ins 19. Jahrhundert zurückdatieren, und vielfältig ergänzt und überlagert werden durch justiziell entwickelte Rechtsgrundsätze.

Tradition und revolutionäre Sozialutopie, diese beiden einander scheinbar ausschlieBenden, zumeist jedoch eigentümlich inspirierenden "Zukunftserinnerungen " an bessere Zeiten bilden die geschichtlichen Ströme, die kompromißhaft gebrochen in das Betricbsrätegesetz von 1920 einfließen.

\section{a) Die sozial-reformerische Tradition der Arbeiterausschüsse}

Der Gesetzgeber konnte schon zu diesem Zeitpunkt auf eine etablierte und weithin akzeptierte Tradition der Arbeitnehmetrepräsentanten im Betrieb zurückgreifen. Sie reicht zurück auf Entwürfe der Frankfurter Paulskirchenversammlung von 1849 zur betrieblichen Interessenvertrctung und Schlichtung durch "Fabrikausschüsse «, "Fa-

3 Während die Entwurfe der sog. „Vredeling-Richtliniens (ABI. I5.11.1980, Nr. C 297/3 und ABI. 12.8. 1983 , Nr.C 217/3) noch an einem einhcitlichen Mitwirkungsmodell orienticrt waren und daran scheiterten, stellt der Entwurf der s. gesellschaftsrechtlichen Richtlinie uber die Struktur der Aktiengesellschaft (ABI. 9.9.1983, C 240/2) den Mitgliedsstaaten verschredene Mitbestımmungsmodelle zur Auswahl, vgl. den Úberblick bei W. Daubler, M. Kitener, K. Lorcher (Hrsg.), Internationale Arbeits- und Sozılordnung, Koln r 990, S. 103 sff.

4 Die "Richtlinic uber die Einsetzung eines Europaischen Betriebsrats oder die Schaifung eınes Verfahrens zur Unterrichtung und Anhorung der Arbettnchmer in gemeinschaftsweit operierenden Unternehmen und Unternehmensgruppen a (ABI. 30.9. 1994, Nr. L.254 S.64) ist am 22.9. 1994 vom Rat der Arbeits-und Sozialminister der i Signaturstaaten - also ohne Großbritannıen - angenommen worden. Erfaßt werden Unternehmen mit mindestens 1000 Arbeitnehmern in den 11 Unterzetchnerlandern und mit jeweils mindestens tso Arbeitnehmern in mindestens 2 EG-Staaten (ohne Großbritannien). Vgl. zu den Details H. Klinkhammer, D. Welslau, in: ArbuR ${ }_{1994}$ S. $326 \mathrm{ff} ., 457$. 
brikräthe« und "Fabrikschiedsgerichte «s. Seit den 7oer Jahren und verstärkt nach I 889 als Reaktion auf den großen Bergarbeiterstreik kam es insbesondere in Großbetrieben verbreitet zur Errichtung freiwilliger Arbeiterausschüsse, die durch dic Novellen zur Reichsgewerbeordnung von $1981^{6}$ und zum preußischen Berggesetz von $1892^{7}$ gesetzlich verankert wurden. Effektive Mitbestimmungsrechte für die obligatorisch gemachten betrieblichen Arbeiter- und Angestelltenausschüsse räumte im Gegenzug zur kriegswirtschaftlichen Verfügung über die Arbeitskräfte erstmals das Gesetz über den vaterländischen Hilfsdienst vom S. 1 2. $1916^{8}$ ein.

Der Erlaß Kaiser Wilhelms des II. vom 4. 2. $1890^{\circ}$ zur gesetzlichen Einrichtung von Arbeiterausschüssen hat dem sozialintegrativen, wirtschaftsfriedlichen Geist dieser Tradition die Richtung gewiesen:

"Für die Pflege des Friedens zwischen Arbeitgebern und Arbeitnehmern sind gesetzliche Bestimmungen über die Formen in Aussicht zu nehmen, in denen die Arbeiter durch Vertreter, welche ibr Vertrauen besitzen, an der Regelung gemeinsamer Angelegenheiten beteiligt und zur Wahrnehmung ibrer Interessen bei Verhandlungen mit den Arbeitgebern und den Organen Meiner Majestät befähigt werden. Durch eine solche Einrichtung ist den Arbeitern der freie und friedliche Austausch ibrer Wünsche und Beschwerden zu ermöglichen und den Staatsbehörden Gelegenheit zu geben, sich über die Verhältnisse der Arbeiter fortlaufend zu unterrichten und mit Letzteren Fühlung zu behalten."

Diese staatliche Initiative zur Entdramatisierung von Arbeitskonflikten durch die Schaffung einer Repräsentationsinstanz der Arbeitnehmer und die Eröffnung betrieblicher Informations- und Kommunikationswege sowie behördlicher Aufsichtsbefugnisse stieß zunächst auf den gemeinsamen Widerstand von Sozialdemokratie, Gewerkschaften und Großindustrie: Erstere sahen in der Förderung der machtpolitisch vom Unternehmer abhängigen Arbeiterausschüsse mit den Worten August Bebcls lcdiglich ein »scheinkonstitutionelles Feigenblatt, mit dem der Fabrikfeudalismus verdeckt werden soll «" . Ihrer "lahmen und zahmen Thätigkeit « sagte Max Schippel weitgehende Wirkungslosigkeit voraus". Wegen des wirtschaftlichen

s Vgl. den Entwurf eincr Gewerbeordnuni; fur das Deutsche Reich vom Februar 18.49, abgedruckt in: T. Blanke, R. Erd, U. Muckenberger, U.Stascheit, Kollektives Arbeitsricht. Quellentexte zur Geschichte des Arbeitsrechts in Deutschland, 1975 , Bd. 1 S. $42 \mathrm{f}$

6 RGBI. 1891, 261 ff., auszugsweise abgedruckt in: T. Blanke u. a., a. a. O., S. $92 \mathrm{ff}$.

7 PrGS 1892, 131 ff.; durch die Novelle dieses Gesetzes vom 14. 7. 1905 , prGS 1905, S. 307 ff., auszugsweise abgedruckt beı T. Blanke u. a., a. a. O. S. 114 ff. wurde erstmals die Errichtung von Arbeiterausschussen beschrankt auf die preußischen Bergbaubetriebe - obligatorisch gemacht.

8 RGBl. $1916 \mathrm{Nr}, 276$, S. 1333 ff. auszugsweise abgedruckt bei T. Blanke u. a., a. a. O. S. $161 \mathrm{ff}$

9 Auszugsweise abgedruckt bei T. Blanke u. a., a. a. O. S. 90 ; ganz parallel lautet die Zweckbestummung der „Ordre, betreffend dıe Errichtung von Vertrauensmannerkonferenzen " auf den Rust Pleßschen Kohlegruben v. 10. 7. 1889 , wieder abgedruckt bei J. Ruckert, W. Friedrich, Betriebliche Arbeiterausschisse in Deutschland, Großbritannien und Frankreich im spaten 19. und fruhen 20. Jahrhundert, Frankfurv/M. 1979. S. ${ }_{2} 3 \mathrm{ff}$.

to Verhandlungen des Reichstages, 8. Legislaturperıode, 4. Band, Berlin 1891, S. 2324.

$11 \mathrm{Vgl}$. dessen Analyse der Funktion der Arbeiterausschusse in: Neue Zeit 1990/91, 1. Band, S. I29ff., auszugsweise abgedruckt in: T. Blanke u. a., a. a. O. S. $86 \mathrm{ff}$; die von Schippel versammelten Argumente bilden bis heute das Grundgerust jener orthodoxen okonomistischen Position, die die Spielraume fur freiheitliche, demokratische und soziale Weiterentwicklungen dcs Arbeitsrechts als außerst gering einschatzt; angesıchts der Dominanz weltwirtschaftlicher Sachzwange gilt ihr zufolge dies nicht nur auf der individualvertraglichen und betrieblichen, sondern ebenso auch auf der tarifvertraglichen und selbst der nationalen und europaischen Ebenc. Die Unternehmer und ihre Verbande werden dem nur zu gerne beipflichten. Gleichwohl zeigt die Geschichte des Arbeitsrechts, daß die von der okonomisehen Logık gezogenen Grenzen so hart und eindeutıg besummbar nicht sund, weil die arbettsrechtichen Normen hhrerseits zum Bestimmungsfaktor okonomisch-sozialer Prozesse werden und diese modifizieren. Hier findet enne komplexe Wechselwirkung statt, auf die beretts Karl Marx bei seiner Analyse der Funktion der Begrenzung der Arbeitszeit fur den Ubergang von der absoluten zur relativen Mehrwertproduktion durch technologische Rationalısierung gernache hat, vgl. Das Kapital, Bd. I (MEW Bd, 23), 197t, S. 225 f. 
"Konkurrenzkrieges« zwischen den Unternehmen sei es strukturell ausgeschlossen, daß sie im Einzelbetrieb Verbesserungen für die Belegschaften erzielen könnten, die ökonomisch zu Buche schlügen. Dies könnte vielmehr allein den branchenweit agierenden und kampffähigen Gewerkschaften gelingen, auf deren Schwächung folglich die Errichtung der Arbeiterausschüsse ziele. Dagegen befürchtete der mächtige Zcntralverband deutscher Industricller, daß vermittelt über dic Arbeiterausschüsse die Gewerkschaften erst richtig in den Betrieben Fuß fassen würden. ${ }^{12}$ Bereits wenige Jahre später war diese ablehnende Haltung der Sozialdemokratie und der Koalitionen aufgrund der mit der betrieblichen Interessenvertretung gemachten praktischen Erfahrungen und ihrem erheblichen Bedeutungszuwachs einer verhaltenen positiven Einstellung gewichen. ${ }^{13}$

\section{b) Der Einfluß der Rätebewegung}

Der entscheidende Impuls zur Fort- und Umbildung dieser sozial-reformerischen Tradition der Arbeirerräte in Richtung auf die Etablierung eines betrieblichen Bürgersratus ging von der Rätebewegung aus. Sie, und nicht etwa die sozialistische Orthodoxic der ctablierten Organisationen der Arbeiterbewegung, entfaltete die politische Sprengkraft, die zum Betriebsräregesetz von 1920 führte - auch wenn es sich hierbei aus der Sicht der Revolutionäre von 1918 um eine der späten und stark verwässerten, ja vergifteten Früchte der Novemberrevolution handelte, wcnn etwa der Abgeordncte der USPD, Dr. Geyer, in der Parlamentsdebatte vom 14. 1. 1920 vom "Gesetz gegen die soziale Revolution" sprach.

Die Rätebewegung hatte auf politischer Ebene zum Sturz der Monarchie und zur Errichtung der parlamentarischen Demokratie geführt. Aber die Revolution vom November 1917 war nicht nur eine politische Bewegung, sondern zugleich - wenn nicht sogar in erster Linie - eine sozialc. Der mit der Niederlage im ersten Weltkrieg einhergehende Zusammenbruch der alten Ordnungsmächte führte an vielen Orten zur spontanen Bildung von Arbeiter- und Soldatenräten. Sie übernahmen Aufgaben der politischen Repräsentation, der Gesetzgebung, Verwaltung, Polizei bis hin zur Demobilmachung und der Lenkung des $W$ irtschaftsprozesses. Auch wenn die Rätcbewegung zu zersplittcrt und vielfältig war, um cine konsistente politische Programmatik zu entwickeln, so zeichnete sich ihre Sozialismuskonzeption doch durch charakteristische Momente aus: Ihre "syndikalistische« Orientierung an eincm basisdemokratischen, alle gesellschaftlichen Bereiche umfassenden Modell der politisch-sozialen Selbstverwaltung und cinem damit verbundenen starken antibürokratischen und antiinstitutionellen Impuls, der sich aus der Kritik an preußischen Obrigkeitsstaat, an Militarismus und Klassenjustiz ebenso wie an der Burgfriedenspolitik von SPD und ADGB angesichts des ersten Weltkrieges, dem strukturellen Opportunismus von bürokratischen Großorganisationen, speiste. Im Gegensatz zum damaligen Aufbau der Gewerkschaften bildete nicht der Beruf, sondern der Betrieb die Organisationsgrundlage der Rätebewegung. Anknüpfend an den Status als Produzenten stand die Aufhebung des betrieblichen K-lerrschaftsverhältnisses, die

I 2 Vgl. J. Rückert, W. Friedrich (Fn. g) S. $21 \mathrm{f}$.

13 Seit 1909 war die sozialdemokratısche Reichstagsfraktıon fur die Einfuhrung obligatorischer Arbeiterausschusse eingetreten, vgl. Sozıale Praxis 1908/9 S.638; vorausgegangen war dem ein Einstellungswandel in den Gewerkschaften, vgl. etwa C. L.egien, Ziele und Mittel der deutschen Gewerkschaftsbewegung, sozialıstische Monatshefte 1900, $109 \mathrm{ff}$; eine 1906 durchgefuhrte Untersuchung des Vereins deutscher Maschinenbauanstalten ergab, daß zwei Drittel der Unternchmen, in denen Arbeiterausschusse bestanden, sich posituv uber ihre Wirkungen aussprachen, vgl. Soziale Praxis tg05/6 S. 351 ; nicht selten erfolgte die Errichtung von Arbetterausschussen tm Gefolge erbitterter Arbeitskampfe und bistweilen wurde ihr Bestand tarifvertraglich abgesichert, vgl. zu den Details J. Ruckert, W. Friedrich, a.a.O. S. $25 \mathrm{ff}$. 
bewußte demokratische Aneignung und kollektive Veränderung des Produktionsprozesses im Zentrum ihrer Ziele und Aktivitäten - die "Eigentumsfrage « war ihr demgegenüber wenn nicht politisch zweit- und historisch nachrangig, so doch jedenfalls nicht die conditio sine qua non einer umwälzenden sozialistischen Strategie. Größer konnte die Differenz zur orthodox-sozialistischen Prämisse vom Primat der Eroberung der staatlich-politischen Macht, um das Privateigentum an Produktionsmittcln aufzuheben, zu verstaatlichen und in planmäßig-bürokratische Kontrolle und Lenkung zu überführen, kaum ausfallen: Wer glaubte, Bürgerrechte und Demokratie im Betrieb sowie die Selbstverwirklichung der Arbeitnehmer als Produzenten ungeachter der fortbestehenden kapitalistischen Eigentumsverhältnissc verwirklichen zu können, saß aus dieser traditionalistischen Sicht einem kapitalen Irrtum über die Härte der ökonomischen Funktionsgesetze und die Schärfe der Klassengegensätze auf. Er war crgriffen von der verhängnisvollen voluntaristischen Illusion einer Überschätzung subjektiver Gestaltbarkeit der bestehenden sozialen Verhältnisse. Einig, und darin licgt die Gemeinsamkeit der konträren sozialistischen Positionen, waren sie sich in dem Glauben an eine prinzipielle Versöhnbarkcit der in der modernen Geselischaft auseinandergetretencn, zu verobjektivierten Systemfunktionen verselbständigten Wertsphären von Wissenschaft, Kunst, Religion, Moral und Politik eine Illusion, die sich nach dem crsten Weltkrieg durch alle sozialen Schichten und allc politischen Lager hindurchzog und pittoresk gesteigert massenhaften Ausdruck fand. ${ }^{14}$

Das politische Schicksal der Rätebewegung war zwar vergleichswcisc rasch, niche zuletzt durch den Einsatz militärischer Gewalt, entschieden. Aber die SPD und die Gewerkschaften untcr Führung des ADGB sahen sich durch die Stärke der Bewegung doch veranlaßt, die Idee einer rätcdemokratischen Umgestaltung und Organisation der Wirtschaft wenigstens partiell aufzugreifen. "s Eines ihrer Rcsultate war die in Artikel ${ }^{6}{ }_{5}$ der Wcimarer Reichsverfassung vorgesehenc, nur in unbedeutenden Ansätzen realisierte Bildung von Arbeiter- und Wirtschaftsräten auf Bezirks- und Reichsebene, durch die die gleichbercchtigte Mitwirkung der Arbeiter und Angestellten zusammon mit den Unternehmern san der Regelung der Lobn-und Arbeitsbedingungen sowie an der gesamten wirtschaftlichen Entwicklung der produktiven Kräfte" herbeigeführt werden sollte. Ein anderes war das Betriebsrätegesetz.

14 Es war die Zeit der Inflationsheilıgen, die auf der Straße, in den Waldern, auf den Marktplatzen, in den Zirkuszelten und den verraucherten Hinterzimmern von Kneipen Deurschland oder die Welt erlosen wollten. Oswald Spenglers, Der Untergang des Abeadlandese, in jenen Jahren sechshundertausendmal verkauft, war der großtheoretische Entwurf, der in tausend kleine Splitter, Weltdeutungen aus dem Geiste von Endzeit und radikalem Neubeginn, zersprang. Fast jede großere Stadt verfügte über einen oder sogar mehrere , Heilander... Die zahlreichen Propheten und Charismatiker jener Jahre sind fast alle millenarisch oder apokalyptisch gestimmt, es sind Irrganger der revolutionaren Erregungen bei Kriegsende, Dezisionisten der Welterneuerung, wild gewordene Metaphysiker und Geschaftemacher auf dem Jahrmarkt der Ideologien und Ersatzreligionen. Wer sich um seine Seriositat sorgte, ging auf Distanz zu dieser Schmuddelszene, doch waren die Übergange durchaus fließend. Dies gilt auch für die politische Szene im engeren Sinne, wo Messianismus und Heilslehren links und rechts ebenfalls uppig gediehen. In den Tagen der Munchener Raterepublik kundigt en von Toller und Muhsam verfaßter Erlaß die Verwandlung der Welt in seine Wiese voll Blumenr an, in der jeder seinen Teil pflucken konne, es wird Ausbeutung, jegliche Hierarchie und juristisches Denken fur ahgeschafft erklart und den Zeitungen befohlen, auf der Titelseite Gedichte von Holderlin oder Schıller neben den neuesten Revolutionsdekreten zu publizieren $\propto$, R. Safranski, Ein Meister aus Deutschland. Heidegger und seine Zeit, 1994, S. $116 f$.

is D Der Gedanke, durch das Betriebsrategesetz ein Ventil fur das räterevolutionare Drangen der Arbeitermassen zu schaffen, durchzicht als leitendes Motiv alle Beratungen des Gesetzes, und dient den herrschenden Gruppen als Begrundung und Entschuldigung, den opponierenden als Vorwurf und Anklage. Es kommt hinzu, daß die Ratebewegung in den politischen Arbeiterraten und den revolutionaren Betriebsràten bereits ihren praktischen Niederschlag gefunden hat, und daB speziell die revolucionaren Betriebsräte, die sich in wichtigen Industriegebieten weitgehend durchgesetzt haben, ein Faktum darstellen, das weder übergangen noch rückgangig gemache werden kann ..., $\mathrm{K}$. Brigl-Matchiaß, Das Betricbsrateproblem, i926, S. 2. 

Konflikt

Mit der ursprünglichen Idee rätedemokratischer Organisation der Produktion hatte es wenig mehr als den Namen gemein: Statt auf Selbstverwaltung durch die Arbeitnehmer setzte es wie bereits Art. 16s der WRV auf konfliktregulierende Zusammenarbeit mit den Unternehmern. So heißt es in $\$ 1$. BRG:

„Zur Wabrnebmung der gemeinsamen wirtschaftlichen Interessen der Arbeitnehmer (Arbeiter und Angestellten) dem Arbeitgeber gegenüber und zur Unterstützung des Arbeitgebers in der Erfüllung der Betriebszwecke sind in allen Betrieben, die in der Regel mindestens zwanzig Arbeitnehmer beschäftigen, Betriebsräte zu errichten. « Deutlich wird diese kooperative Grundtendenz auch in $\$ 66 \mathrm{BRG}$, wonach der Betriebsrat die Aufgabe hat

1 . in Betrieben mit wirtschaftlichen Zwecken die Betriebsleitung durch Rat zu unterstützen, um dadurch mit ibr für einen möglichst hohen Stand und für möglichst hobe Wirtschaftlichkeit der Betriebsleistungen zu sorgen;... 3. den Betrieb vor Erschütterungen $z$ u bewabren, insbesondere vorbehaltlich der Befugnisse der wirtschaftlichen Vereinigungen der Arbeiter und Angestellten (\$8), bei Streitigkeiten des Betriebsrats, der Arbeitnehmerschaft, einer Gruppe oder eines ibrer Teile mit dem Arbeitgeber, wenn durch Verbandlungen keine Einigung zu erzielen ist, den Schlichtungsausschuß oder eine vereinbarte Einigungs- oder Schiedsstelle anzurufen; . . 6. das Einvernehmen innerhalb der Arbeitnebmerschaft sowie zwischen ibr und dem Arbeitgeber zu fördern.....

In welchen Maße dieses Betriebsrätegesetz von 1920 strukturbildend für die deutsche Betriebsverfassung bis heute geworden ist, läßt sich daran ablesen, daß bereits damals alle Grundprinzipien, die diese bis heute auszeichnen, im Kern angelegt sind. Dies gilt insbesondere für

- die Einführung der dualen Struktur der Interessenvertretung durch die Verselbständigung der Betriebsräte gegenüber den Gewerkschaften

- das Primat der Tarifvertragsparteien zur Regelung der Arbeitsbeziehungen ( $\$ \$ 8$, 66 Ziff. 3,78 Nr. 2 BRG)

- das Gebot der kooperativen Zusammenarbeit mit der Betriebslcitung zum Wohle des Betriebes (Betriebsgemeinschaft)

- die Trennung von betrieblicher und wirtschaftlicher Mitwirkung bzw. -bestimmung (und damit des juristischen Betriebs- vom Unternehmensbegriff, $\$ \$ 6$, is ff., 7० BRG)

- die unterschiedliche Legitimationsbasis von Betriebsräten gegenüber Gewerkschaften (demokratische Repräsentation auf der Grundlage der Betriebszugehörigkeit durch allgemeine Wahlen gegenüber demokratischer Legitimation auf der Grundlage freiwilliger Verbandsmitgliedschaft)

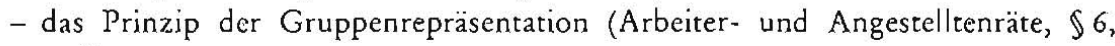
is ff., I9 BRG)

- die institutionelle Verselbständigung der Betricbsrätc gegenübcr der Arbeitnchmerschaft (Tragung der Kosten der Betriebsratsarbeit durch den Arbeitgeber; ausdrückliches Verbot der Erhebung und Leistung von Beiträgen der Arbeitnehmer für »irgendwelche Zwecke der Betriebsvertretungen «, $\$ \$ 36,37 \mathrm{BRG}$; feste Amtsperiode ohne Rückberufungsrecht durch die Arbeitnehmer)

- die Verrechtlichung und Prozeduralisierung des sozialen Konfliktfeldes Betrieb nach Maßgabe enumerativ aufgeführter Mitwirkungsrechte des Betriebsrats und Verfahrensbestimmungen ( $\$ \$ 66,78 \mathrm{ff} . \mathrm{BRG}$ ) 
- seine Pazifizierung (Streikverbot, $\$ 66$ Abs. 3) und Entpolirisierung (Beschränkung der zulässigen Themen von Betriebsversammlungen, $\$ 48$ BRG)

- die eingeschränkte Anwendung des Gesetzes in Tendenzbetrieben $(\$ 67 \mathrm{BRG}$; anders als heute galt das BRG jedoch auch im Bereich der öffentlichen Verwaltung und war die Errichtung von Bctriebsräten nicht fakultativ, sondern zwingend vorgesehen, $\$ \$ 1,9 \mathrm{BRG}$ )

- die unangetastete Leitungszuständigkeit des Arbeitgebers $(\$ 69$ BRG: •Die Ausfübrung der gemeinsam mit der Betriebsleitung gefaßten Beschlüsse übernimmt die Betriebsleitung. Ein Eingriff in die Betriebsleitung durch selbständige Anordnungen stebt dem Betriebsrat nicht $z u($ ).

Diese bis heute dominanten Strukturmerkmale der deutschen Betriebsverfassung haben, wie gesagt, mit den expliziten Absichten und Zielen der Rätebewegung so gut wic nichts gemein. Selbst wenn man mit Ernst Fraenkel dem Betriebsrätegesetz unterstellt, daß »es das Mittel sein sollte, die Arbeiterschaft aktiv an der Gesamtwirtschaft zu beteiligen«, ist er doch Realist genug zu konstatieren, daß es insowcit wegen der "Verkennung der ökonomischen Entwicklungstendenzen * durch den Gesetzgeber "weitgehend ein Versager geworden (ist) ${ }^{16}$. Die zentrale Bcdeutung des Bctricbsrätegesetzes sieht $\mathrm{cr}$ in seincr sozialpsychologischen Wirkung: «Das Betriebsrätegesetz hat sich als Integrationsmittel des Proletariats im Sinne Rudolf Smends bewährt. «"

Mochte das Betriebsrätegesetz aus der Sicht der revolutionären Räte von 1918 auch als "großer Betrug " erscheinen, ein Verdikt, dem sich auch Kar] Korsch ${ }^{18}$ anschloß, so wäre es doch falsch, damit zugleich in der von Fraenkel hervorgehobenen sozialintegrativen Wirkung sciner koopcrativen Struktur nichts als ein ideologisches Täuschungsmanöver über die "wirklichen « Intercssen der Arbeitnehmer und die Reichweite ihrer Durchsetzbarkeit zu sehen und diese Wirkung folglich für definitiv abqualifiziert zu erachten. Karl Korsch selbst bestcht im Gegenteil darauf, daß durch dieses Gesetz die Struktur der industriellen Beziehungen eine neue Qualität erhalten hat. Der »gewerbliche Konstitutionalismus « werde die rein privatrechtlich-individualvertragliche Auffassung des Arbeitsverhältnisses zugunsten einer kollektiven ("proletarischen «) »sozialrechtlichen « Konzeption verdrängen, die »das Arbeitsverhältnis als ein lediglich auf die gesellschaftliche Zusammenarbeit gegründetes Gemeinschaftsverhältnis ansieht «' ${ }^{19}$ - ein Gemeinschaftsverhältnis, dessen volle Entfaltung aus seiner Sicht freilich dic Aufhebung der bestehenden Eigentumsverfassung voraussetzt und in der gesetzlich verordneten Variante der kapitalistischen "Betricbsgemeinschaft « einen irreführenden, ideologischen Ausdruck erhalten hat.

Dicse paradigmatisch für die Einschätzung des Betriebsrätegesetzes durch die arbeitsrechtliche Linke in Weimar stehenden Auffassungen von Fraenkel und Korsch bezeichnen bei aller Ambivalenz sehr gut die spezifisch neue Qualität und Wirkung dieses Gesetzes. Alle seine zentralen Strukturmerkmale sind präzis auf einen gemeinsamen Zweck zugeschnitten: Sie zielen auf die Schaffung eines Modells der regulierten Konfliktaustragung und -bewältigung sowie der ökonomisch-sozialen Zusam-

16 (Fn. 1) S. 98.

17 A.a.O.S. 107.

I8 Zir. nach E. Gerlach, Vorwort zum Nachdruck von *Arbeitsrecht für Betriebsratex, (Fn. I) S. 7.

19 (Fn. I) S. 149. Als Inbegriff solcher "sozialrechelicher Normen* betrachtet er insbesondere das Betriebsrátegesetz. und erwartet von deren Weiterentwicklung etwa den Fortbestand der Arbeitsverhaltnisse und der Betriebsratsamter im Streik - eben jene skollektive Betrachrungsweise a, zu der der Große Senac des AG mit seinem Grundsatzbeschluß vom 28. 1. 195s, AP Nr. 1 zu Ar. 9 GG - Arbeitskampf, gefunden hat. Der Ertrag dieser rechtskonstruktiven Wende für das BAG bestand freilich darin, daß dadurch die Moglichkeit zur Entwicklung einer justizıellen Feinmechanik zur Regulierung von Arbeitskämpfen eroffnet wurde. Das soziale Freiheitsrecht des Streikrechts wurde umgebildet zu emem funktionalen Mechanismus im System der Tarifautonomie. 
menarbeit im Betrieb und führen mit der »sozialrechtlichen« Ausgestaltung der Arbeitsverfassung einen juristischen Paradigmenwechsel herbei, demzufolge sie nicht länger eine beziehungslose Summe individueller Vertragsverhältnisse darstellt, sondern dic Qualität eines sozialen Kooperationsverhältnisses erhält.

Die erfolgreiche Durchsetzung "ziviler Umgangsformen " in der betrieblichen Sphäre, dic von der zunächst rechtlich weitgehend ungebundenen wirtschaftlichen und sozialen Dominanz (juristisch: dem Eigentums-, Direktions- und Kündigungsrecht) des Arbeitgebers, dem Fabrikdiktat, gekennzeichnet ist, ist ein äußerst komplexer und fast unwahrscheinlich zu nennender Vorgang der "Befriedung" dieses wohl konfliktträchtigsten Feldes der Gesellschaft. Er ist nach wie vor unabgeschlossen und höchst voraussetzungsvoll. Zu seinen externen Bedingungen zählt gewiß eine Periode politischer Stabilität und wirtschaftlicher Prosperität, dic freilich ihrerseits nicht zuletzt auf der Entfaltung koopcrativer Strukturen der wirtschaftlichen Verbände beruht. Damit setzt er zu allererst von beiden "Konfliktpartnern", Arbeitgebern wie Arbeitnchmern, voraus, daß sie nicht - im Sinne von Carl Schmitts Begriff des Politischen, der zeitdiagnostisch verstanden erschreckenden Realitätsgehalt bekommen solltc - wechselseitig auf ihre Vernichtung ausgehen.

\section{Das Scheitern des Weimarer Versuchs zur Demokratisierung von Staat, Gesellschaft, Wirtschaft und Betrieb}

Genau diese elementare Voraussetzung der Anerkennung der wechselseitigen Existenzberechtigung und Funktionsnotwendigkeit aber konnte zum Zeitpunkt der Entstehung des Betriebsrätcgesetzes und den Jahren danach nicht gemacht werden. Politik und Wirtschaft waren sowohl weltanschaulich-ideologisch wie politisch-organisatorisch derart miteinander identifiziert, $d a ß$ an eine selbständige, autonome Austragung von Interessengegensätzen in der jeweiligen Sozialsphäre allenfalls in den kurzen Prosperitätsphasen der Weimarer Zeit zu denken war. Mit der krisenhaften Zuspitzung ökonomischer Konflikte erodierte sogleich das Spektrum politischer Gemeinsamkeiten, die das Fundament der Demokratie bilden. Nicht zufällig interpretieren Otto Kahn-Freund ${ }^{20}$ und Ernst Fraenke ${ }^{21}$ jeden Angriff auf die Funktionsmechanismen der sozialen Selbstverwaltung in Gestalt der zentralen Normen des kollektiven Arbeitsrechts als Angriff auf die Republik. Dics war, wie sich zum bösen Ende herausstellen sollte, keine falsche Dramatisierung.

Das Betriebsrätegesetz von 1920 war demnach - wie die Weimarer Reichsverfassung und ihr Projekt einer Demokratisierung von Staat und Wirtschaft insgesamt - auf schwankenden Boden gebaut. Es mußte sich die Verhältnisse jenes wechsclseitigen

20 Das soziale Ideal des Reıchsarbeitsgerichts, in: T. Ramm (Hrsg., Fn. 1) S. 149 ff. Kahn-Freund beschließt seine Analyse der arbeitsgerichtlichen Rechtsprechung mit der Warnung: ${ }^{\prime}$ Die politischen Parteien als Träger der aktıven politischen Kräfte des Volkes erhalten in unserer Gesellschaft ihr sozıales Gepräge und damit ihre polttische Wegrichtung weitgehend durch die sozialen Verbande, die hinter ihnen stehen. Nimmt man diesen sozialen Verbanden den Kampfcharakter, sucht man an die Stelle des sozialen Kampfes die Herrschaft des hoheren Beamtentums zu setzen, so hat man mit diesem System sozialer Diktatur die soziologischen und psychologischen Voraussetzungen auch der politischen Diktatur geschaffen. Die Tatsache, daß sowohl die faschistische als auch die kommunistische Diktatur den Arbeitskampf verboten hat, ist gewiß kein Zufall.e A. a. O. S. $209 \mathrm{f}$.

21 "Deshalb zielt der Angriff gegen die Unabdingbarkett des Tarifvertrages nicht einmal in erster Linie auf eine Verlagerung der wirtschaftlichen und sozıalen Kräfteverteilung, er zielt der deutschen Republik mitten ins Herz... So wird der Kampf um die Aufrechterhaltung des Tarffvertrages zugleich ein Kampf um die Fortexistenz der Republik «, in: Die politısche Bedeutung des Arbeitsrechts, in T. Ramm (Hrsg.), a. a. O.S. 247. Trotz aller Dramatik der Veranderungen auch im gegenwartigen Arbeitsrecht: Von solcher Dramaturgie ist die Bundesrepublik zum Gluck weit entfernt. 
Vertrauens, die die Voraussetzung für eine funktionierende kooperative Betriebsverfassung sind, erst schaffen, um so wirken zu können, wie gesetzlich vorgesehen. Aber dazu sollte es in der kurzen Zeit der Weimarer Republik nur in Ansätzen kommen. Als die Nationalsozialisten mit tatkräftiger Unterstützung des schwerindustriellen Großkapitals gegen die Republik putschten, waren ihre Diktatur die Strukturen des kollektiven Arbeitsrechts ein besonderer Dorn im Auge: Gewährleisten sie doch die Autonomie sozialer Organisation und Willensbildung und die Strukturen einer begrenzten Konfliktaustragung, die dokumentierten, daß trot $\angle$ aller völkisch-rassischen Einheits- und Gleichhcitspropaganda nach wie vor ticfgreifendo Intcressengegensätze die Gesellschaft durchzogen. Nach der selbst für die NS-Führer überraschend widerstandslos hingenommenen Zerschlagung der Gewerkschaften, zu der die Besetzung der Gewerkschaftshäuser am 2. Mai 1933 das offizielle Fanal gab, erset $\iota t e$ das "Gesetz zur Ordnung der nationalen Arbeit « vom 20. I. 1934 die Betriebsräte durch "Vertrauensmänner«. Ihre Liste wurde vom Unternehmer als "Betriebsführer aufgestellt. Mitzuentscheiden hatten sie nichts: "Der Führer des Betriebes entscheidet der Gefolgschaft gegenüber in allen betrieblichen Angelegenheiten, soweit sic durch dieses Gesetz geregelt werden « ( $\$ 2$ Abs. x AOG).

\section{Die Etablierung der zweifachen Struktur der Interessenvertretung durch Gewerkschaften und Betriebsräte: Konkurrenz oder Kooperation?}

Nicht zufällig gcht die Festlcgung auf eine sozial kooperative, konfliktregulierende Form der betrieblichen Mitbestimmung durch das Betriebsrätegesetz einher mit der Errichtung der dualen Form der Interessenvertretung der Arbeitnehmer durch Gewerkschaften einerseits, Bctriebsräte andererseits. Erst diese institutionelle Verselbständigung der betrieblichen Interessenrepräsentation bot dic Chance zur Entwicklung einer Praxis, die sich stärker an den Strukturcn der betrieblichen Kooperation als an der konfliktorientierten, klassenkämpferischen Programmatik der dominierenden Gewerkschaften orientierte. Diese Struktur gibt das Grundmuster der industriellen Bcziehungen in Deutschland bis heute ab und wird, wie ohnc großes Risiko prognostiziert werden kann, auch das Modell der künftigen europäischen Arbeitsbezichungen prägen:

- Danach sind die Gewerkschaften zuständig für die Vertretung der Arbcitnehmer in ihrer Rolle als Anbieter auf dem Arbeitsmarkt. Durch die Tarifverträgc mit den Arbeitgebern legen sie mit gleichsam gesetzlicher, zwingend-normativer Wirkung die "Verkaufsbedingungen « der Arbeitskraft fest und regeln im Detail die zcntralen Elcmente der Austauschbeziehungen von Arbeit und Kapital.

- Die Betriebsräte sind dagegen zuständig für die Vertretung der Arbeitnehmcr in ihrer Rolle als Betriebsangebörige, also an dem Ort, an dem sie ihre Arbeitsleistung erbringen: Dabei liegt der Schwerpunkt ihrer Befugnisse auf der Mitwirkung in personellen und sozialen Angelcgenheiten, während er in wirtschaftlichen wie produktionstechnischen Fragen gering ausgeprägt ist.

- Hier setzt als weitere Säule der Mitbestimmung die Vertretung der Arbeitnehmer als wirtschaftliche Produzenten durch dic Arbeitnehmerrepräscntanten im Aufsichtsrat an, so daß man auch von einer dreigliedrigen, strialen « Struktur der Intercssenvertretung sprechen könnte. Die wirtschaftliche Mitbestimmung ist durch das Mitbestimmungsgesetz von 1976 jedoch auf große Kapitalgescllschaften mit mehr als 2000 Arbeitnehmern beschränkt und damit nicht annähernd 
flächendeckend ausgebaut wie die beiden anderen Dimensionen der Mitbestimmung.

Die Vertretung der sozialen Interessen der Arbeitnehmer kann sich niemals auf eine dieser Ebenen allein beschränken. Sie muß vielmehr den Versuch machen, an die unterschiedlichen Rollen, die die Arbeitnchmer als Arbcitsmarktteilnehmer ( Verkäufer« der Arbeitskraft, auch als Arbeitslose), als Betriebsangehörige (Mitglieder der Betriebsorganisation) und wirtschaftliche Produzenten (sowie ggf, als Konsumenten) einnehmen, anzuknüpfen und die sich daraus ergebenden, durchaus spezifischen Interessen aufzugreifen und möglichst effektiv zu Geltung zu bringen. Hierüber besteht im Grundsatz Einigkeit. Umstritten jedoch war und ist in der Geschichte der Arbeiter- und Gewerkschaftsbewegung in Iiuropa bis heute, ob die Aufspaltung derkollektiven Interessenvertretung der Arbeitnehmer auf verschiedene Institutionen und Akteure (Gewerkschaften, Betriebsräte, Aufsichtsratsvertreter) zur Stärkung oder zur Schwächung der Interessenvertretung insgesamt führt. Ob sich Gewerkschaften und Betriebsräte gegenseitig eher behindern oder in ihren jeweiligen Zuständigkeiten und Funktionen kooperativ ergänzen, läßt sich nicht abstrakt beurteilen, sondern hängt von vielerlei Faktoren ab: Der wirtschaftlichen Lage, der Repräsentanz der Gewerkschaften im Betrieb, der gewerkschaftsorganisatorischen Einbindung der Betriebsräte, der politischen und tariflichen Strategie der Gewerkschaften und der gesetzlichen Ausgestaltung der Funktion und Rolle der Betricbsräte. Aber zweifellos hat das Betriebsrätegesetz von 1920 die Voraussetzungen dafür, daß es zu einer wechselseitigen Verselbständigung beider Formen der Interessenvertretung kommen kann, wenn nicht geschaffen, so doch verbessert - und dies ganz bewußt und gezielt.

"Teile und herrsche $\propto$ ist ein bewährter Grundsatz der Strategie der Machterhaltung. Er erklärt die Skepsis, die die Gewerkschaften gegenüber einer Verselbständigung der betrieblichen Interessenvertretung hegen und macht verständlich, warum bis heute etwa die britischen und französischen Modelle des kollektiven Arbeitsrechts auch auf Betriebsebene allein eine Mitbestimmung durch gewerkschaftliche Repräsentanten kennen. Andererseits ist deutlich, daß die Interessen der Arbeitnehmer in Abhängigkeit von ihrer jeweiligen Rolle durchaus unterschiedlich, ja sogar gegensätzlich sind oder jedenfalls sein können. So sind sie als Arbeitsmarktteilnehmer (zumindest objektiv) an der Verringerung der Konkurrenz und folglich an einheitlichen, standardisierten Lohn- und Gehaltstarifen interessiert, als Betricbsangehörige und Produzenten hingegen an cinem Einkommen, welches der betrieblichen Produktivität und ihrer spezifischen individuellen Leistungsfähigkeit entspricht, als Konsumenten an möglichst billigen Produkten und niedrigen Produktionskosten, zu denen wesentlich die Löhne zählen, als lebenslang Lohnabhängige an umfassender sozialer Absicherung für die Risiken der Arbeitnehmerexistenz, an Qualifikation und Weiterbildung sowie umfassendem Gesundheits- und Umweltschutz. Und als (vorübergehend) Arbeitslose an einer Senkung der Zugangsbarrieren zum Arbeitsplatz und damit u. U. sogar an einem Abbau arbeitsrechtlicher Schutzbestimmungen. 
Gegenwärtig hat es den Anschein, daß soziale wie ökonomische und rechtlich-politische Faktoren zu einer weiteren Ausdifferenzierung dieser divergierenden, an die jeweilige Arbeitnehmerrolle gebundenen Interessenlagen führen. Dafür sprechen ganz unterschiedliche Entwicklungen wie die Tendenz zur Individualisierung von Lebenslagen, zur Differenzierung von Bedürfnissen und Interessen zwischen den Arbeitnehmern und innerhalb der unterschiedlichen Stadien einer Erwerbsbiographie, die zunehmende Integration von Frauen ins Erwerbsleben und ihr Anspruch auf Gleichstellung, die Auswirkungen der Flexibilisierung von Produktion, Organisation, Absatz und Vertrieb auf dic Gestaltungsmöglichkeiten der Arbeitsprozesse und die Anforderungen an die Arbeitnehmer, der Anstieg und die Verstetigung der Massenarbeitslosigkeit und der Abbau rechtlicher wie sozialer Schutzstandards. Dieser ökosoziale Strukturwandel hat im deutschen Arbeitsrecht im vergangenen Jahrzehnt jedoch nicht einfach nur politische Initiativen zur Deregulierung ausgelöst. ${ }^{22}$ Vielmehr hat er vor allem die Gewichtung zwischen den verschiedenen Rollen der Arbeitnehmerexistenz in dem Sinne verschoben, daß die Bedingungen kollektiver Interessenvertretung um so schwieriger werden, je allgemeiner und abstrakter sie ansetzt. ${ }^{23}$ Die Folge ist eine erhebliche Aufwcrtung der Ebene der betrieblichen gegenüber der tariflich-gewerkschaftlichen Mitbestimmung, ${ }^{24}$ die Stärkung der individucllen Autonomie und der Persönlichkcitsrechte des Arbeitnehmers, ${ }^{25}$ die nicht nur verbale Anerkennung der Gleichberechrigung der Frauen ${ }^{26}$ sowie die Berücksichtigung auch der Interessen der Arbeitslosen (und von Entlassung Bedrohten) etwa in den Arbeitszeittarifverträgen.

Die wachsende objektive und subjektive Relevanz der individuellen und betrieblichen Entfaltungschancen der Arbeitnehmer stellt die Gewcrkschaften, dic gewohnt sind - und deren Funktion es ist - auf der Makroebene des Marktes zu agieren, vor erheblich neue Herausforderungen. Auch wo sie wie in Deutschland aufgrund der Entwicklung einer eigenständigen betrieblichen Interessenvertretung von der widersprüchlichen Aufgabe entlastct sind, zu ciner einheitlichen Intcressenvertretung in allen ökonomisch-sozialen Dimensionen der Arbeitnehmerexistenz zu finden, wird die norwendige Vermitrlung zwischen dicsen verschicdenen Rollen deutlich schwieriger.

Ob es den Gewerkschaften gelingt, ähnlich wie bisher durch eine aktivc Betriebs-, Vertrauensleute- und Betriebsrätearbeit politisch-programmatisch und organisato-

22 Vgl. T. Blanke, Flexibilisierung und Deregulierung: Modernisierung ohne Alternative?, in: W. Daubler, M. Bobke, K. Kehrmann (Hrsg.), Arbeit und Recht. Festschrift fur Albert Gnade, 1992, S. 2 sff.

23 Nach einer aktuellen Umfrage des SPIEGEL antworten auf die Frage: „Erfullen die Gewerkschaften noch ihren Zweck oder mußten sie sich entsprechend der wirtschaftlichen Entwicklung reformieren?. $74 \%$ mit *müßten sich reformieren . Nur $21 \%$ sind der Meinung, daß sie noch ihren Zweck erfüllen, vgl. Nr. 5 v. 30. 1. 1995, S. 84 .

24 Dies wirft eine Fulle rechtskonstruktiver Probleme auf, wcil dic Betricbsautonomie gegenüber der Tarıfautonomie als gesetzlich nachrangig ausgestaltet isc. Vgl. F.-J.Säcker, H.Octker, Grundlagen und Grenzen der Tarifautonomie, 1992; Th. Baumann, Die Delegation tariflicher Rechtsetzungsbefugnisse, 1992; Th. Kreuder, Recheliche Aspektc esner Tarifpolitik im Umbruch, in: T. Blanke, E. Schmidt (Hrsg.), Tarifpolitik im Umbruch, r995, S. $37 \mathrm{ff}$.

2s Vgl. S.Simitis, in: Zacher, Simitis, Kübler, Hopt, Teubner, Verrechtlichung von Wirtschaft, Arbeic und sozialer Solidaritat, I 984 ; dgl., Die Loi Le Chaelier: Bemerkungen zur Geschichte und moglichen Wicderenideckung des Indivituums, in: KJ H. 2/1989, S. 157 ff.; dgl., Die vcrordnete Sprachlosigkeit: Das Arbeitsverhaltnis als Kommunikationsbarricte, in: Festschroft fur H. Simon, 1987, S. 329 ff.; H. Matchies, U. Muckenberger u. a., Arbeit 2000, a.a.O., S. 70 ff., 258 tt.; U. Wendeling-Schroder, Autunomic im Arbeitsrecht. Moglichkeiten und (irenzen eigenverantworclichen Ilandelns in der abhangigen Arbeit, 1994.

26 Vgl. S. Raasch, Fraucnquoten und Männerrechte, 1991. 
risch zusammenzuhaltcn, was zwar zusammengehört, abcr institutioncll und rechtlich verselbständigt ist und sichtbar auseinanderdriftet, ist nicht ausgemacht. Optimistisch stimmen die bemerkenswerten Innovationen in den letztjährigen Tarifrunden und der in ihnen erbrachte Beweis der Mobilisierbarkcit von Solidaritätspotentialen, die längst totgesagt wurden. Andererseits ist die Tatsache nicht zu überschen, daß auch dic bundesdeutschen Gewerkschaften von der Krise eingeholt werden, die nicht zufällig bereits in den $800 r$ Jahren insbesondere dic britischen und die französischen Gewerkschaften in aller Dramatik ereilt hat: Drastisch sinkende Mitgliederzahlen und schwindende Finanzmittel sind die Indizien für eine ticfgreifende Krise der Repräsentation. Sie macht die Neuorientierung der Gewerkschaften zur Überlebensfrage. ${ }^{27}$ Dabei wird endgültig eine »heilige Kuh « aus dem ideologischen Traditionsbestand der Arbeiterbewegung geschlachtet werden müssen, deren Gang zum Altar bereits mit dem Betriebsrätegesetz von 1920 begann: Die Vorstellung von der Notwendigkeit und Möglichkeit einer einheitlichen organisatorischen und programmatischen Interessenrepräsentation der Arbeitnehmer in all ihren unterschiedlichen wirtschaftlichen und sozialen Rollen durch die Gewerkschaften.

\section{Perspektive: Die Entfaltung eines betrieblichen Arbeitskampfrechts?}

Die Etablierung der dualen Struktur der Interessenvertretung durch das Betriebsrätegesetz von 1920 war aus dieser heutigen Perspektive von erstaunlicher Weitsicht. Einer Weitsicht, die weder einen individuellen noch einen kollektiven Urheber hatte, sondern aus einer komplexen Mischung höchst unterschiedlicher Interessen und Absichten, Traditionen, Strukturen und Strategien resultierte und die überdies als Innovation von historischer Tragweite damals kaum zu erkennen war. Wenn nicht alles täuscht, dann ist dic Verselbständigung dieser betrieblichen Ebene der kollektiven Mitbestimmung einer der Garanten für die im europäischen Maßstab erstaunlich große Stabilität des Systcms der industriellen Beziehungen in Deutschland und scines Arbeitsrechts. Sie fungiert als ein gleichsam hydraulischer Mechanismus der Problemverlagerung, der es erlaubt, Rigiditäten infolge zu hoher Zentralisierung durch Delegation auf cine untere, sachnähere Ebene abzubauen und flexibler Regulierung zuzuführen.

Die Karriere der Betriebsebene, erst recht des Individualarbeitsrechts, zur Regulierung der Arbeitsbeziehungen ist rechtlich und sozial jedoch alles anderc als unproblematisch. Das auch vom Bundesverfassungsgericht erkannte und anerkannte Faktum der wstrukturellen Unterlegenheit des einzelnen Arbeitnehmrs $\alpha^{28}$ ist durch spezifische ökonomische Besonderheiten des Arbeitsmarktes, insbesondere dic fehlende Exklusivität der Eigentumsrechte, ${ }^{29}$ bedingt. Es setzt die Möglichkeit, im Arbeitsrecht durch individuellc Vertragsaushandlung einen angemessenen, beiderseitig gerechten Interessensausgleich herbeiführen zu können, im Normalfall definitive Grenzen. Diese zu überwinden, ist die genuine, inzwischen um eine Fülle weiterer gesellschaftlicher Schutzinteressen erweiterte moralische und soziale Funktion des Arbeitsrechts. Hierbei nahmen und nehmen die Gewerkschaften nach deutschem Recht insofern einc privilegierte Rolle cin, als sie im Kontext des tariflichen Verhand-

27 Darauf geht naher U. Muckenberger, Akcuelle Herausforderungen an die Tarifpolitik, in diesem Heft S. $26 \mathrm{ff}$. cin.

28 BVerfG v. 26.6. 199t, ArbuR 1991, S. 30 ff.

29 Vgl. E. Dorndorf, Mehr Individualfresheit im Arbejtsreche? in: W. Daubler, M. Bobke, K. Kehrmann (Hrsg., Fn. 22) S. $39 \mathrm{ff}$. 
lungssystems über das Monopol der kollcktiven Kampffähigkeit verfügen. Die sichtbare Funktionseinbuße dieser Regulierungsebene der Arbeitsbeziehungen kann durch einen waffenlosen, allein auf informelle Möglichkeiten kollektiven Agierens und die Kraft von Argumenten gestützten Betricbsrat kaum angemessen kompensiert werden. Vor die Demokratie im Betrieb hat das bürgerliche Recht das Eigentum, das Arbeitsrecht die Kündigung gesetzt. Demokratisierung der betrieblichen Sphäre sctzt daher - auch dies lehrt die Geschichte des Betriebsrätegesetzes von 1920 - Konfliktualität im Sinne erhöhter Konflikt- und Risikobereitschaft einerseits, ${ }^{30}$ erhöhter institutionell und rechtlich gesicherter Konfliktfähigkeit andererseits voraus. Der Bedeutungszuwachs der Betriebsebene für die Gestaltung der Arbeitsbezichungen wirft daher das Problem der Entwicklung eines betrieblichen Arbeitskampfrechts auf. Die Konturen eines solchen Rechts können viclfältig sein - etwa als kollektives Zurückbehaltungsrecht, Neuauflage betriebsnaher Tarifpolitik und Vordringen von Firmentarifverträgen oder, was ein neuerlicher Quantensprung der Betriebsverfassung wäre, einer Arbeitskampffähigkeit der Betricbsräte. Auch wenn es derzeit nicht den Anschein hat: Die Tage werden kommen, an denen es eingefordert wird.

30 Vgl. eindringlich wird diese Voraussetzung effektuver Mitbestummung belegt in der empirischen Untersuchung von $\mathrm{H}$. Kotthoff, Betriebsrate und Burgerstatus. Wandel und Kontinuitat betrieblicher Mitbesummung, 1994 